\title{
Design of Cloud Monitor System for Fire Detection Based on Video Streaming
}

\author{
Haiyan Li, Lihui
}

\begin{abstract}
Considering the limits such as slower reaction time, larger scale layout cost of traditional smoke sensors, temperature sensors and other hardware technology. In this paper, a cloud monitor system based on video stream is designed. The design of the system hardware and software platform will be introduced. The system uses TI's TMS320DM642(as DM642) and Freescale's i.MX27 as CPU. DM642 has powerful data processing capabilities. i.MX27 has rich interface and powerful video compression capabilities. The system consists of three subsystems: video processing system (DM642 as core), video transmission system (i.MX27 as core) and display system based on C\# .
\end{abstract}

Index Terms-Video-based fire detection,TMS320DM642, i.MX27, Flame image processing

\section{INTRODUCTION}

Fire detection techniques are currently smoke type, temperature-sensitive, sensitive type, etc. These techniques are generally perceived to detect fire through a variety of sensors to sense such as temperature, brightness or other features. But In large space buildings, these fire detection methods can not detect fire earlier in order to reduce the loss of life and property. For video-based fire detection method, when the fire broke out, the camera can capture the video information of fire immediately, identify the fire through a certain image algorithm processing, and send alarm at once. The method can achieve early detection of fire. It is possible to reduce the fire detection error rate by improving the image algorithm, to achieve fast and accurate fire detection. So researchers pay more and more attention on fire detection system based on video stream.

For these purposes, we start to design a fire detection system based on DSP and ARM. The system uses TI's TMS320DM642 and Freescale's i.MX27 as CPU. A display system based on C\# language to display real-time monitoring video and send alarm to administrators.

\section{OVERALl DESIGN OF SYSTEM}

In Fig.1, it is shown the framework of system. The system collects the visible and infrared video stream in monitoring zone through the camera, the collected video is analog signal, than converted into an identifiable digital signal stream through the video process system based on DM642. DM642 has multiple video ports(called VP0 2) and can accept multiple video streams at the same time, so the same monitoring area zone can have multiple cameras.

DSP preprocesses the video signal by noise filtering and fire image feature detection to determine whether there is a

Haiyan Li master's degree at Nanjing University of Science and Technology, 2014.9-2017.4, Jiangsu Province, China

Lihui doctor's degree at Nanjing University of Science and Technology, 2016. fire. The built-in hardware alarm device can complete the preliminary field alarm. The processed video signal is transmitted to the video transmission system based on i.MX27. i.MX27 uses the embedded H.264 module to compress the video stream, and transmits the compressed data to the cloud platform for further processing and alarm.

The system is mainly divided into video processing system with DM642 as the core, video transmission system with i.MX27 as the core, and cloud platform for display and alarm. DM642 with complex instruction set is responsible for video acquisition and processing, and ARM926EJ-S core with simplified instruction set runs Linux operating system for H.264 video coding compression and network transmission. The system board integrates i.MX27 and DM642 on one board, which has great advantages in stability.

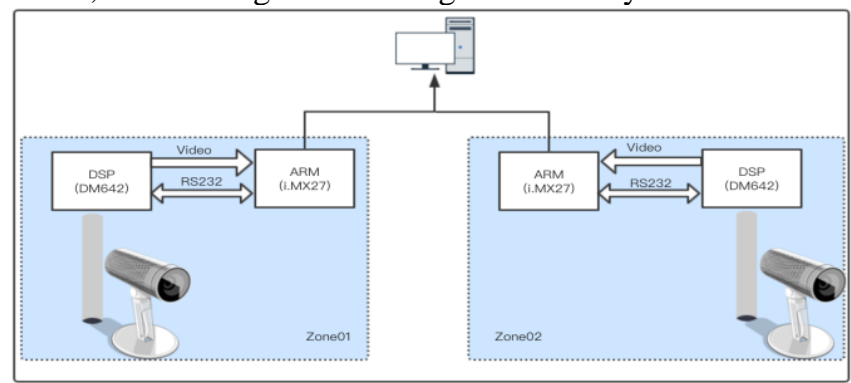

Fig.1. System design diagram.

\section{VIDEO PROCESS SYSTEM}

Video collect and algorithm processing are completed by the video process module with DM642 as the core. As shown in Fig.2, the analog video stream collected by the camera is decoded by the video decoding chip 5150AM1 and then transmitted into DM642 (Note: actually that the video signal is transmitted into the peripheral memory chip SDRAM of DM642 through DMA). The program detects whether there is flame over the image frame. After the detection, it passes through the video port VP0 of DM642 to the video coding chip SAA7121 to code video. Than it can be transmitted to the display or the i.MX27 module for compression transmission. DM642 peripheral extended CAN bus as alarm signal line.

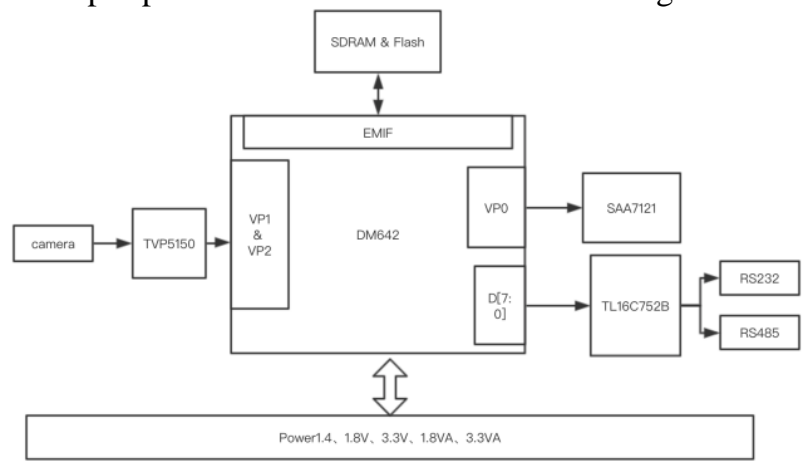

Fig. 2. Video process system. 


\section{VIDEO TRANSMISSION SYSTEM}

The system expands NAND flash and DDR SDRAM memory as Fig.3 shown, DDR SDRAM is mainly used to store large capacity video data, and NAND flash is mainly used to store programs. The system has one analog video input and one analog video output. The input analog video signal is converted into digital video signal by video decoding chip and then transmitted to CPU. The digital video signal processed by CPU is converted into analog signal by video encoding chip and output to display module. The i.MX27 uses the internal H.264 encoding module to compress the digital video signal, and upload the compressed video data to the upper computer for display through the fast Ethernet chip. System has a serial port, which is used to boot load, file system mount and write the custom program.

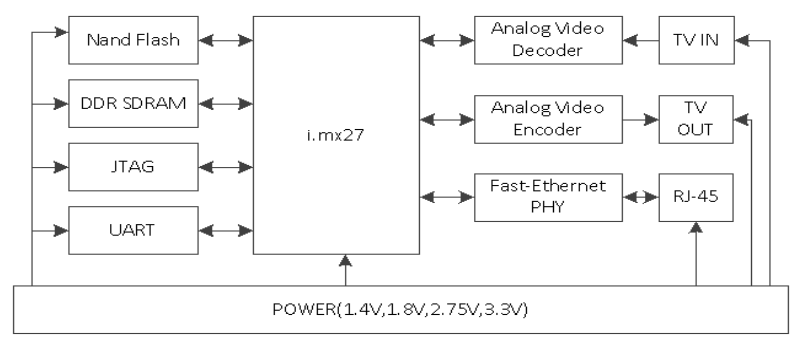

Fig. 3. Video transmission framework.

\section{VIDEO DISPLAY AND ALARM}

After the video stream is processed by DM642, it will be delivered to the i.MX27 module which is responsible for compressing the video stream. The i.MX27 has its own hardware compression module. Here, it is specified to compress the video stream in H.264 format. The compressed video stream is transmitted to the upper computer monitoring platform through the network cable, and the monitoring platform receives the H.264 code stream compressed from i.MX27, decodes and plays it like Fig.4. RTP and RTCP protocol are used for video transmission, which is improved based on UDP protocol. On the basis of ensuring real-time video transmission, the transmission quality is improved by collecting confirmation signals from both ends.

This paper focuses on the hardware system development. Based on this platform, some practical engineering algorithms can be verified. In order to facilitate the development and debugging of the relevant parameter threshold, a computer program which can communicate with DSP serial port is built. Through the computer program, the system can control the working mode, calibrate and set the parameter threshold.

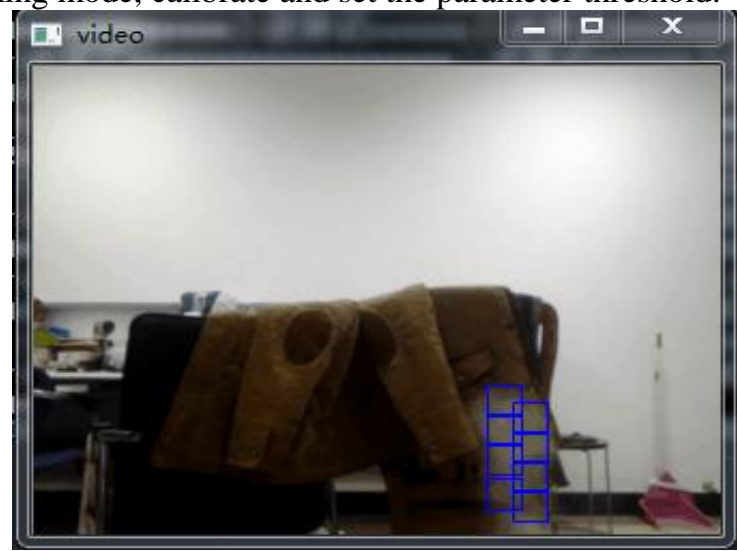

Fig. 4. Video display.

\section{CONCLUSION}

A fire detector based on DM642 and i.MX27 is designed and implemented. The hardware design and video display alarm software are introduced. The system is mainly designed for large-scale space such as weapon warehouse, shopping mall and other places where the fire source needs to be strictly controlled.

\section{REFERENCES}

[1] Benxi Wang. Research on fire detection technology based on image processing [D]. Donghua University.2006(in Chinese).

[2] Wang Tao, Liu Yuan, Xie Zhen-ping. Flutter Analysis Based VideoSmoke Detection [J]. Journal of Electronics \& InformationTechnology, 2011, 3 (5): 1024-1029 (in Chinese).

[3] Ko, ByoungChul and Park, JunOh and Nam, Jae-Yeal.Spatiotemporal bag-of-features for early wildfire smoke

[4] Hidenori Maruta,Akihiro Nakamura,Fujio Kurokawa. Smokedetection in open areas with texture analysis and support vectormachines[J]. IEEJ Trans Elec Electron Eng, 2012,7S1:. pagesS59-S70.

[5] A. Ochoa-Brito et al. "Improvement of a Video Smoke Detection Based on Accumulative Motion OrientationModel" Proceedings of the Electronics, Robotics and Automotive Mechanics Conference (CERMA), pp. 126-130,Cuernavaca, Morelos, Mexico, 15-18 November 2011..

[6] Rui Tong.Design of remote video surveillance system and smoke detection system based on S3C2440 [D].Chengdu University of Technology, 2012(in Chinese).

[7] Laifa Fang,Hongyong Yuan.Using DSP to realize the automatic fire alarm system [J].Electronic Technique,2000,(10):57-59(in Chinese).

[8] Haicai Sun,Xin Bi.Design of forest fire detection system based on embedded processor platform [J].Computer Engineering and Design , 2010,31(6):1184-1187.

[9] Nobuyuki,Fujiwara,Kenji.Terada. Extraction of a Smoke Region Using Fractal Cording. Jntenational Symposium on Communication and Information Technologies.2004.October:26-29.

[10] I.Kopilovic, B.Vagvolgyi,andT.Sziranyi. Application of panoramic annular lens for motion analysis tasks: surveillance and smoke detection. Proceedings of 15 th International Conference on Pattern Recognition,2000,Volume 4,3-7 Sept.2000.pp.714-717.

[11] Surapong Surit, Watchara Chatwiriya "Forest Fire Smoke Detection in Video Based on Digital Image ProcessingApproach with Static and Dynamic Characteristic Analysis," cnsi, pp.35-39, 2011 First ACIS/JNU InternationalConference on Computers, Networks, Systems and IndustrialEngineering, 2011.

[12] H. Maruta, Y. Kato, A. Nakamura, F. Kurokawa, "Smoke detection in open areas using its texture features and timeseries properties", IEEE International Symposium on Industrial Electronics, ISIE 2009, pp. 1904-1908.

[13] B. U. Toreyin. Fire detection algorithms using multimodal signaland image analysis [D]. Ankara: Cankaya University. Departmentof Electronic and Communication Engineering, 2009.

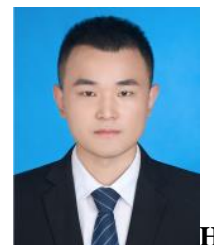

Haiyan Li master's degree at Nanjing University of Science and Technology, 2014.9-2017.4, Jiangsu Province, China. The main research direction is video image processing based on DSP. Now working at Netease Inc China, He current research interests include image processing and kubernetes cluster management.

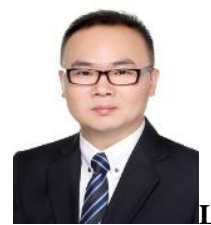

ihui doctor's degree at Nanjing University of Science and Technology, 2016.4-now, Jiangsu Province, China. Main research directions: Internet of things(IoT), wireless sensor network ,networked control system, distributed control system and intelligent control. 